\title{
Ausência de Prejuízo no Reconhecimento de Expressões Faciais entre Indivíduos com Parkinson
}

\author{
Adriana Manso Melchiades Nozima* \\ Bibiane Demos \\ Wânia Cristina de Souza \\ Universidade de Brasília, Brasília, DF, Brasil
}

\begin{abstract}
RESUMO - Entre os sintomas não motores da doença de Parkinson, dificuldades no reconhecimento de expressões faciais emocionais vêm sendo amplamente discutidas, pois as áreas cerebrais relacionadas a tal habilidade podem estar afetadas na doença. Este estudo investigou, em idosos, o reconhecimento das seis expressões emocionais faciais consideradas universais por meio do instrumento Teste de Percepção Emocional de Faces, em que participantes executam uma tarefa de reconhecimento de expressões emocionais faciais. Participaram 41 indivíduos com idade média de 64,9 anos, 27 homens e 14 mulheres. Não foi observada significativa dificuldade no reconhecimento de nenhuma das expressões emocionais por parte dos parkinsonianos. Tal resultado pode indicar a necessidade do desenvolvimento de instrumentos e técnicas mais adequadas para esse tipo de investigação na população brasileira.
\end{abstract}

Palavras-chave: doença de Parkinson, expressões faciais, emoção, reconhecimento.

\section{Lack of Impairment of Facial Expression Recognition among Individuals with Parkinson Disease}

\begin{abstract}
Among the non-motor symptoms of Parkinson's disease, impairment in the recognition of emotional facial expressions has been widely discussed, as the disease may affect brain areas related to this skill. The current study has investigated, among elderly people, the recognition of the six emotional face expressions considered universal using the instrument Test of Emotional Perception of Faces, where participants performed an emotional facial recognition task. Forty-one participants with an average age of 64.9 years were enrolled to this study, 27 men and 14 women. No significant impairment in the recognition of emotional face expression was observed among the parkinsonians. This result may indicate the need to develop more adequate tools and techniques able to investigate this issue in the Brazilian population.
\end{abstract}

Keywords: Parkinson's disease, facial expression, emotion, recognition.

A doença de Parkinson (DP) é a segunda doença degenerativa mais comum na atualidade, atrás apenas da doença de Alzheimer (Xie, Meng, Xiao, Zhang, \& Zhang, 2016). É mais comum entre idosos e conhecida por suas marcantes características motoras e não motoras. Sua incidência é consideravelmente maior com o aumento da idade, porém estudos recentes indicam que localidade geográfica e sexo também podem contribuir na distribuição da doença ao redor do globo (Pringsheim, Jette, Frolkis, \& Steeves, 2014). Entre 70 e 79 anos de idade, a incidência é maior na América do Norte, Europa e Austrália, assim como, entre 50 e 59 anos, a prevalência da doença é em homens (Pringsheim et al., 2014).

Atualmente, sintomas não motores têm sido fortemente associados a DP. Entre esses, alterações cognitivas e emocionais (Alonso-Recio, Martín-Plasencia, LoechesAlonso, \& Serrano-Rodríguez, 2014). Características como enfraquecimento cognitivo, depressão, psicose, distúrbios do sono e dor têm tido grande reflexo nas avaliações de

* Endereço para correspondência: Campus Universitário, Darcy Ribeiro, ICC, Ala Sul, Asa Norte, Brasília, DF, Brasil. CEP: 70.910-900.

E-mail: adriana.melchiades@gmail.com qualidade de vida, índices de institucionalização e impacto socioeconômico (Chaudhuri, Healy, \& Schapira, 2006; Xie, et al., 2016).

Em função da existência de manifestações cognitivas e comportamentais na DP, existe grande interesse na investigação de um possível prejuízo no reconhecimento de expressões emocionais faciais por parte desses pacientes, uma vez que a doença pode trazer alterações em áreas cerebrais estratégicas para a identificação da expressão facial, como a ínsula, a amígdala, estriado ventral e o córtex pré-frontal (áreas pré-límbicas, giro do cíngulo anterior, córtex insular e órbito-frontal) (Janvin, Aarsland, Larsen, \& Hugdahl, 2003; Mentis \& Delalot, 2005; Chaudhuri et al., 2006; Assogna, Pontieri, Caltagirone, \& Spalletta, 2008; Rosso, Nicaretta, \& Mattos 2008).

\section{Expressão das Emoções}

A expressão de emoções tem função social e papel decisivo no processo de interação humana. Do ponto de vista evolucionista, a face tem importância fundamental para os 
seres humanos, pois tem a função de demonstrar emoções por meio do movimento dos músculos. Ekman e Friesen (1971), ao realizarem uma série de replicações dos experimentos de Darwin (1882/2000) sobre as expressões faciais, chegaram a um padrão morfológico esperado para cada emoção. Apesar de ser um tema ainda controverso na literatura (Russel, 1994; Jack, Blais, Scherpers, Schyns, \& Caldara, 2009), mesmo em diferentes culturas, com elementos distintos envolvidos na reação emocional, uma grande proporção de pesquisadores trata as emoções básicas descritas nos experimentos de Darwin como universais. São elas: medo, surpresa, raiva, nojo, tristeza/pesar e felicidade/alegria (Ekman \& Oster, 1979; Ekman, 1993; Fernández-Dols, 2013; Fernãndez-Dols \& Crivelli, 2013).

O reconhecimento das expressões faciais emocionais está diretamente relacionado ao comportamento social não verbal e, consequentemente, à adaptação do ser humano a diferentes contextos do desenvolvimento (De Souza, Feitosa, Eifuku, Tamura, \& Ono, 2008). Clark, Neargarder e Cronin-Golomb (2010) identificaram que uma empobrecida habilidade em funções executivas se relaciona a fixações mais prolongadas em pacientes com Parkinson. Seus achados indicaram uma relação específica entre o déficit de reconhecimento facial emocional e o escaneamento visual de expressões emocionais. Tal achado pode indicar que o comportamento de escaneamento visual durante uma tarefa de categorização emocional é guiado por diferentes processos perceptuais e estratégias cognitivas na DP (Clark, et al. , 2010).

\section{Reconhecimento das Expressões Emocionais Faciais na Doença de Parkinson.}

Já há alguns anos, dificuldades no reconhecimento de expressões emocionais têm sido vastamente relatadas na DP (Ariatti, Benuzzi, \& Nichelli, 2008; Borod et al., 1990; Dara, Monetta, \& Pell, 2008; Schröder et al., 2006; Simons, Ellgring, \& Pasqualini, 2003). Entretanto, a literatura ainda não é clara, uma vez que alguns estudos apontam para o prejuízo de tal habilidade (Breitenstein, Daum, \& Ackermann, 1998; Dujardin et al., 2004; Herrera, Cuetos, \& RodriguezFerreiro, 2011; Yip, Lee, Ho, Tsang, \& Li, 2003), e outros para sua manutenção (Adolphs, Schul, \& Tranel, 1998; Borod et al., 1990; Cohen, Gagne, Hess, \& Pourcher, 2010; Pell \& Leonard, 2005). Há ainda estudos que indicam prejuízos no reconhecimento de emoções específicas (Assogna et al., 2008; Kan, Kawamura, Hasegawa, Mochizuki, \& Nakamura, 2002; Suzuki, Hoshino, Shigemasu, \& Kawamura, 2006), assim como diversas são as justificativas para as possíveis variações (Assogna et al., 2008; Dujardin et al., 2004; Gray \& Tickle-Degnen, 2010; Herrera et al., 2011; Perón, Dondoine, Le Jeune, Grandjean, \& 2012; Suzuki et al., 2006; Yip et al., 2003).

Pacientes no estágio inicial da DP frequentemente manifestam disfunção no gânglio basal, estrutura importante no controle motor e participante do processamento cerebral de emoções. Em decorrência disso, podem apresentar enfraquecimento no reconhecimento emocional de expressões faciais (Breitenstein et al., 1998; Cools, Miyakawa, Sheridan,
\& D’Esposito, 2010; Jacobs, Shuren, Bowers, \& Heilman, 1995).

Do ponto de vista do processamento cognitivo, uma das instâncias necessárias para o reconhecimento de estímulos emocionais é a memória de trabalho (Phillips, Channon, Tunstall, Hedenstrom, \& Lyons, 2008). Na DP, prejuízos têm sido observados em relação a essa habilidade, especialmente no que se refere a tarefas viso-espaciais (Costa et al., 2003; Lee et al., 2010; Possin, Filoteo, Song, \& Salmon, 2008; Stoffers, Berendse, Deijen, \& Wolters, 2003).

Martínez-Coral et al. (2010) pesquisaram a existência de prejuízos no reconhecimento de faces emocionais em pacientes parkinsonianos, os quais apresentavam ou não apatia. Os pacientes com apatia tiveram pior escore no reconhecimento de medo, raiva e tristeza. Os parkinsonianos não apáticos, bem como os participantes controles, não obtiveram diferenças significativas. Lawrence, Calder, McGowan e Grasby (2002) observaram que apenas a expressão de raiva foi deficitariamente reconhecida por parkinsonianos. Em sua interpretação, tal evidência aponta para a participação da circuitaria neural dopaminérgica no reconhecimento dessa emoção, uma vez que o Parkinson se caracteriza pela perda do tecido nigro-estriatal. Avançando nessa investigação, Lawrence, Goerendt e Brooks (2007) observaram que pacientes com suspensão abrupta da terapia de reposição dopaminérgica apresentaram prejuízo significativo no reconhecimento da raiva em relação a pacientes parkinsonianos medicados ou controles, sugerindo a existência de uma relação entre a interrupção da terapia de reposição de dopamina e o declínio no reconhecimento da expressão de raiva. Anteriormente, fortalecendo essses achados, os mesmos autores já haviam demonstrado que o próprio tratamento de bloqueio dopaminérgico em voluntários saudáveis ocasionou prejuízo transitório no reconhecimento dessa mesma emoção (Lawrence et al., 2007).

Também Dujardin et al. (2004) investiram na investigação da relação entre DP e o reconhecimento das expressões emocionais. Testaram o reconhecimento de expressões emocionais faciais em 18 parkinsonianos (em estágio precoce da doença e não medicados) e 18 controles. Os parkinsonianos apresentaram enfraquecimento significativo na decodificação das emoções de raiva, tristeza e nojo em relação ao controle (Dujardin et al., 2004). Sprengelmeyer et al. (2003) observaram, inclusive, que a percepção do nojo em pacientes parkinsonianos não medicados foi consistentemente pior em relação aos medicados, apontando, segundo os autores, para uma possível participação da circuitaria de neurônios dopaminérgicos também no reconhecimento de expressões de nojo.

Em outros estudos acerca das expressões faciais emocionais na DP, o reconhecimento de surpresa mostrouse prejudicado em parkinsonianos com comprometimento cerebral esquerdo, e de raiva naqueles com comprometimento cerebral direito (Clark, Neargarder, \& Cronin-Golomb, 2008). Já no estudo de Yoshimura, Kawamura, Masaoka e Homma (2005), a expressão de surpresa ativou regiões cerebrais como o giro fusiforme, o giro temporal superior direito, o giro parahipocampal, o córtex cingulado e o cerebelo. 
Segundo Ariatti et al. (2008), as expressões de medo e tristeza foram as menos reconhecidas pelos parkinsonianos, enquanto as outras (alegria, raiva, nojo, surpresa) obtiveram índices de reconhecimento similares ao grupo controle. No estudo das autoras, curiosamente os pacientes com maior alteração motora no hemicorpo direito não reconheceram medo e os prejudicados à esquerda não reconheceram tristeza.

$\mathrm{O}$ reconhecimento de expressões faciais emocionais também foi investigado por Yip et al. (2003), em parkinsonianos com comprometimento motor bilateral. Segundo seus achados, parkinsonianos com comprometimento motor bilateral apresentaram pior reconhecimento de todas as seis emoções universais quando comparados com seus pares saudáveis, especialmente em relação ao medo e à tristeza. Já pacientes afetados motoramente apenas à direita demonstraram dificuldades mais amenas no reconhecimento das emoções. Nesse caso, os resultados apontaram para maior dificuldade na identificação da tristeza, seguida do nojo e nenhuma dificuldade no processamento de faces alegres (Sprengelmeyer et. al., 2003; Yip et al., 2003).

Wieser et al. (2006) também verificaram déficits na discriminação da emoção na DP. Sua conclusão veio por meio de uma tarefa em que 54 fotos emocionais caracterizadas como de alta/baixa indução de emoção (positiva/negativa) foram apresentadas aos pacientes, revelando que os parkinsonianos tiveram diminuição nas respostas emocionais em relação ao controle. Observou-se, pois, que indivíduos com DP podem apresentar maior dificuldade no reconhecimento das expressões emocionais da face, o que se deve às alterações estruturais cerebrais decorrentes da doença, uso de medicação e estágio da doença.

Em virtude da relevância do reconhecimento das expressões emocionais nos seres humanos, incluindo-se aqui pacientes com DP, o objetivo da presente pesquisa foi verificar a habilidade de reconhecimento de expressões emocionais faciais entre indivíduos com Parkinson na população brasileira, por meio especificamente do Teste de Percepção de Expressões Faciais (TEPEF; Ladislau, 2010; Ladislau, Guimarães, \& De Souza, 2015). Hipotetiza-se aqui que participantes com DP terão maior dificuldade no reconhecimento de expressões emocionais quando comparados a participantes sem DP. Diversos estudos já foram realizados abrangendo essa temática, porém, pouco se tem investigado a população brasileira quanto a isso, o que torna esta pesquisa de particular relevância. Aprofundar o conhecimento sobre processos humanos básicos e recorrentes da vida diária em relação à população brasileira pode não apenas contribuir para esse eixo temático, como também vir a oferecer maior expertise a profissionais de saúde e ciência que lidam com o mesmo.

Por ter-se utilizado um novo software instrumental proosto recentemente pela Universidade de Brasília (ainda não disponibilizado comercialmente), o TEPEF, esta pesquisa teve ainda como intento contribuir para a averiguação de sua sensibilidade e confiabilidade para examinar o tema proposto.

\section{Método}

\section{Participantes}

O grupo de pacientes foi composto de 19 idosos diagnosticados com DP, sendo 13 homens e 6 mulheres. A idade variou dos 54 aos 80 anos $(M=63,58)$. Em relação à escolaridade, 15 tinham nível superior, dois, nível médio, um, nível fundamental completo e um, nível fundamental incompleto.

O grupo controle foi composto de 22 idosos saudáveis, convidados a participar do estudo a partir de um grupo de atividade física voltado para idosos, sendo 14 homens e 8 mulheres. A faixa etária do grupo foi de 55 a 80 anos $(M=66,23)$. Quanto à escolaridade, 21 tinham nível superior completo e um, ensino médio.

Presença de alterações cognitivas ou depressão foram considerados critérios de exclusão do estudo. Assim, o Mini Exame do Estado Mental (MEEM; Folstein, Folstein, \& McHugh,1975), com ponto de corte em 23 pontos, e o teste Matrizes Progressivas de Raven, para mensuração do nível intelectual, sendo pontuações entre 25 e 75 consideradas indicativas de inteligência mediana (Raven,1997), foram aplicados a todos os participantes para avaliação dos aspectos cognitivos. A Escala Beck de Depressão foi utilizada para avaliação de humor, com pontuação menor que 10 pontos indicando a presença de depressão (Beck, Steer, \& Brown,1996).

Também se realizou uma entrevista de anamnese inicial e a aplicação da Escala Hoehn e Yahr para verificação do estágio motor da doença (Hoehn \& Yahr, 2001). A maioria dos participantes apresentaram bom desempenho motor, sendo que aqueles com maior comprometimento motor recebiam auxílio da pesquisadora conforme sua necessidade.

Em relação ao resultado descritivo das variáveis relacionadas à DP (tempo de diagnóstico, uso de medicação específica, escala motora da doença e lado motor acometido), o grupo experimental caracterizou-se principalmente por pacientes apresentando o diagnóstico há mais de dois anos e menos de nove anos (68,43\%). A maioria dos pacientes $(89,47 \%)$ faz uso de medicação para controle da DP (variados tipos e dosagens). Já a escala motora (Hoehn \&Yahr, 2001) indicou que $47,37 \%$ apresentam prejuízo motor unilateral, conforme pode ser melhor observado na tabela 1 .

Verificou-se correlação significativa $(p<0,05)$ entre idade e teste intelectual (Raven), assim como entre a escala motora da doença e o teste intelectual. Isso sugere que, quanto mais idade ou comprometimento motor, maiores são as chances de incidência de um nível intelectual mediano (III-).

Por meio do teste chi-quadrado, verificaram-se as variáveis sexo $(p=0,75)$, escolaridade $(p=0,372)$ e teste intelectual $(p=0,79)$, mostrando homogeneidade entre os grupos. A variável idade $(p=0,271)$ também se mostrou homogeneamente distribuída entre grupos. 


\section{Procedimentos:}

Posteriormente a esses instrumentos iniciais, cada participante era avaliado em relação a sua habilidade de reconhecimento de expressões faciais emocionais. Para tanto, utilizou-se o software TEPEF (Ladislau, 2010; Ladislau et al., 2015). Tal software foi desenvolvido e anteriormente utilizado em pacientes portadores da doença de Alzheimer (Ladislau et al., 2015) e Síndrome de Down (Santana, de Souza, \& Feitosa, 2014), contendo 35 expressões faciais, distribuídas entre as emoções básicas consideradas universais

Tabela 1. Caracterização dos grupos controle e experimental

\begin{tabular}{|c|c|c|c|c|c|c|}
\hline \multirow{2}{*}{ Grupo Experimental } & \multicolumn{2}{|c|}{ Masculino } & \multicolumn{2}{|c|}{ Feminino } & \multicolumn{2}{|c|}{ Total } \\
\hline & Absoluto & $\%$ & Absoluto & $\%$ & Absoluto & $\%$ \\
\hline Idade Média & 64,15 & 100,00 & 62,33 & 100,00 & 63,58 & 100,00 \\
\hline Total & 13 & 100,00 & 6 & 100,00 & 19 & 100,00 \\
\hline \multicolumn{7}{|l|}{ Escolaridade } \\
\hline Fundamental Incompleto & 0 & 0,00 & 1 & 16,67 & 1 & 5,26 \\
\hline Fundamental Completo & 1 & 4,69 & 0 & 0,00 & 1 & 5,26 \\
\hline Médio Completo & 1 & 7,69 & 1 & 16,67 & 2 & 10,53 \\
\hline Superior Completo & 11 & 84,62 & 4 & 66,67 & 15 & 78,95 \\
\hline Total & 13 & 100,00 & 62,33 & 100,00 & 19 & 100,00 \\
\hline \multicolumn{7}{|l|}{ Tempo de diagnóstico } \\
\hline Entre 1 e 2 anos & 2 & 15,38 & 0 & 0,00 & 2 & 10,53 \\
\hline Entre 2 e 5 anos & 2 & 15,38 & 5 & 83,33 & 7 & 36,84 \\
\hline Entre 5 e 10 anos & 5 & 38,46 & 1 & 16,67 & 6 & 31,58 \\
\hline Mais que 10 anos & 4 & 30,77 & 0 & 0,00 & 4 & 21,05 \\
\hline Total & 13 & 100,00 & 6 & 100,00 & 19 & 100,00 \\
\hline \multicolumn{7}{|l|}{ Medicação Parkinson } \\
\hline Não utiliza medicação & 0 & 0,00 & 2 & 33,33 & 2 & 10,53 \\
\hline Precursos do paminérgico+agonista & 13 & 100,00 & 4 & 66,67 & 17 & 47,37 \\
\hline Total & 13 & 100,00 & 6 & 100,00 & 19 & 100,00 \\
\hline \multicolumn{7}{|l|}{ Escala Hoehn Yahr } \\
\hline Unilateral & 1 & 7,69 & 1 & 16,67 & 2 & 10,53 \\
\hline Unilateral e axial & 6 & 46,15 & 3 & 50,00 & 9 & 47,37 \\
\hline Bilateral sem déficit de equilíbrio & 0 & 0,00 & 2 & 33,33 & 2 & 10,53 \\
\hline Bilateral leve com recuperação & 1 & 7,69 & 0 & 0,00 & 1 & 5,26 \\
\hline Bilaterial leve e moderada & 4 & 30,77 & 0 & 0,00 & 4 & 21,05 \\
\hline Incapacidade grave & 1 & 7,69 & 0 & 0,00 & 1 & 5,26 \\
\hline Total & 13 & 100,00 & 6 & 100,00 & 19 & 100,00 \\
\hline \multicolumn{7}{|l|}{ Lado acometido } \\
\hline Esquerdo & 6 & 46,15 & 4 & 66,67 & 10 & 52,63 \\
\hline Direito & 7 & 53,85 & 2 & 33,33 & 9 & 47,37 \\
\hline Total & 13 & 100,00 & 6 & 100,00 & 19 & 100,00 \\
\hline \multirow{2}{*}{ Grupo controle } & \multicolumn{2}{|c|}{ Masculino } & \multicolumn{2}{|c|}{ Feminino } & \multicolumn{2}{|c|}{ Total } \\
\hline & Absoluto & $\%$ & Absoluto & $\%$ & Absoluto & $\%$ \\
\hline Idade Média & 66,21 & 100,00 & 66,25 & 100,00 & 66,23 & 100,00 \\
\hline Total & 14 & 100,00 & 8 & 100,00 & 22 & 100,00 \\
\hline \multicolumn{7}{|l|}{ Escolaridade } \\
\hline Fundamental Incompleto & 0 & 0,00 & 0 & 0,00 & 0 & 0,00 \\
\hline Fundamental Completo & 0 & 0,00 & 0 & 0,00 & 0 & 0,00 \\
\hline Médio Completo & 0 & 0,00 & 1 & 12,50 & 1 & 4,55 \\
\hline Superior Completo & 14 & 100,00 & 7 & 87,50 & 21 & 95,45 \\
\hline Total & 14 & 100,00 & 8 & 100,00 & 22 & 100,00 \\
\hline
\end{tabular}


(alegria, tristeza, raiva, surpresa, nojo, medo). Um dos estímulos expressando nojo foi excluído em decorrência de apresentar menor qualidade na padronização em relação ao restante, restando, assim, seis estímulos para cada emoção, exceto nojo que contém cinco estímulos. Os estímulos eram coloridos (fotos) e de alta definição, contendo atores e atrizes expressando cada uma das emoções. Foram apresentados semi-randomicamente por duas vezes aos participantes. A apresentação era semi-randômica para evitar que dois estímulos referentes à mesma emoção fossem apresentados consecutivamente. Os estímulos foram, ainda, apresentados por duas vezes para evitar respostas ao acaso.

Para apresentação, utilizou-se um computador com monitor de LCD de 15 ", onde os estímulos nas dimensões de $9 \times 12 \mathrm{~cm}$, o equivalente a aproximadamente 11.8 graus de ângulo visual, eram centralizados. Juntamente com cada estímulo, apresentavam-se ícones com os nomes das seis expressões emocionais faciais, além do ícone "Não sei". Cada participante deveria observar o estímulo apresentado e escolher uma das alternativas como resposta, sendo que apenas uma delas poderia ser considerada correta.

Durante a avaliação, o computador era posicionado 45 $\mathrm{cm}$ à frente do participante. A pesquisadora posicionava-se à direita do mesmo. A cada aplicação, era verificado se a angulação do monitor proporcionava uma boa visão ao participante. Não havia tempo específico para apresentação dos estímulos, porém, nos casos em que o participante demorava mais que aproximadamente 15 segundos a responder, era-lhe perguntado qual a alternativa mais adequada para aquela expressão. Caso não conseguisse definir sua resposta, ele era solicitado diretamente a indicar uma entre as opções de resposta oferecidas. Por tratar-se de pacientes idosos, uma das preocupações da pesquisa era evitar o cansaço na execução da tarefa. Assim, caso o participante realmente não soubesse indicar alguma resposta, o mesmo era sondado a respeito da escolha da opção "Não sei". Apesar dessa opção ter sido escolhida em menos que 1,7\% das respostas entre os participantes do grupo experimental e menos que $1 \%$ das respostas do controle, a condução do estudo deveria ter observado que esse procedimento traria o risco de impactar a coleta sistemática dos dados. Na medida em que o participante era sondado a respeito da resposta "não sei" após longo tempo de observação do estímulo, é possível que essa sondagem tenha influenciado a resposta. Dessa forma, a metodologia mais adequada teria sido respeitar-se a dúvida do participante por meio da escolha da alternativa "não sei" ou adotar-se um delineamento de escolha forçada. Por tratar-se de participantes com potencial prejuízo motor, o cursor do mouse ficava sob o comando da pesquisadora, que clicava na opção de resposta verbalizada pelo paciente e mantinha o cursor imediatamente abaixo da resposta anterior, buscando evitar indução da resposta seguinte.

As sessões eram agendadas nas residências dos participantes, conforme horário de maior conveniência para os mesmos, tendo a duração aproximada de 1 hora e 30 minutos, a depender do desempenho e agilidade dos próprios participantes. Cada visita iniciava-se com a verificação de um ambiente calmo, bem iluminado, onde houvesse uma mesa e cadeiras. Foi solicitada a ausência de terceiros além do participante e pesquisador no ambiente de coleta, assim como quaisquer eletrônicos ou fontes de possível distração.

Anteriormente à realização deste estudo, foi conduzido um estudo piloto com os estímulos integrantes do TEPEF, com intuito de assegurar o reconhecimento de todas as expressões emocionais expressas por atores por parte da população padrão. A partir do piloto, foram selecionados para integrar o grupo de estímulos para este estudo apenas aqueles com índice de reconhecimento superior a $60 \%$. Todos os procedimentos deste estudo foram submetidos e aprovados pelo Comitê de Ética da Faculdade de Ciências da Saúde da Universidade de Brasília anteriormente a sua realização.

\section{Resultados}

O desempenho dos grupos no reconhecimento de expressões emocionais é descrito na Tabela 1. Conforme pode ser observado na Figura 1, a única expressão totalmente reconhecida por ambos os grupos foi a de alegria $(100 \%)$, enquanto que o medo obteve o menor índice de reconhecimento (49\% no grupo experimental e $37 \%$ no grupo controle). As expressões de tristeza $(85 \%$ grupo experimental e $83 \%$ no grupo controle) e nojo ( $87 \%$ grupo experimental e $82 \%$ no grupo controle) também foram mais bem reconhecidas pelos parkinsonianos. Já as emoções de surpresa ( $82 \%$ no grupo experimental e $90 \%$ no grupo controle) e raiva ( $81 \%$ no grupo experimental e $87 \%$ no grupo controle) foram menos reconhecidas pelo grupo experimental, obtendo melhores percentuais de reconhecimento entre o grupo controle. Entretanto, conforme análise estatística não-paramétrica realizada pelo teste de Mann Whitney, nenhuma dessas diferenças mostrou-se significativa (adotada significância estatística de $p=0,05$ ).

\section{Discussão}

A expressão mais reconhecida foi a de alegria e a menos reconhecida foi a de medo. Apesar de esse achado ser pertinente com a literatura relacionada ao reconhecimento de expressões emocionais, o presente trabalho não encontrou relação estatística significativa entre as variáveis do estudo. A

Índice de Acurácia no Reconhecimeto de Expressões Emocionais

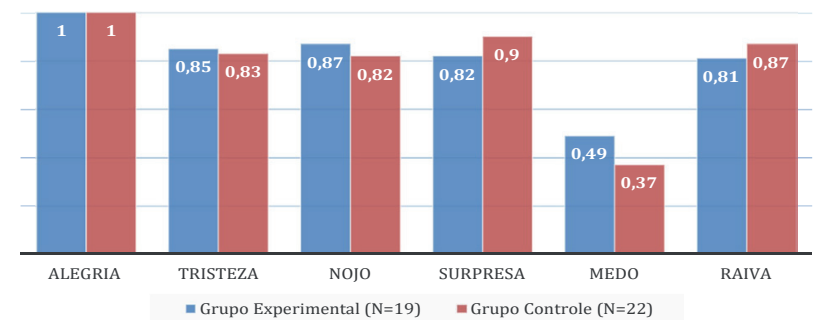

Figura 1. Comparação entre os grupos experimental e controle quanto ao índice de reconhecimento de expressões faciais emocionais entre os grupos $(N=41)$, sendo 1 equivalente a um reconhecimento de $100 \%$. 
primeira explicação plausível para este resultado é o próprio instrumento utilizado para a investigação proposta.

O TEPEF já havia sido utilizado anteriormente em pesquisas com diferentes grupos clínicos, tendo encontrado significativas diferenças na habilidade de reconhecer expressões emocionais entre idosos com Alzheimer (Ladislau et al., 2015) e Síndrome de Down (Santana et al., 2014). Entretanto, em ambos os casos citados, os participantes envolvidos nas pesquisas apresentavam alterações do funcionamento cognitivo, o que não aconteceu na presente pesquisa. A ausência de um resultado significativo pode ser indício de que participantes com melhor perfil cognitivo, porém lentificação/declínio motor, como é o caso deste estudo, tenham se adaptado diferentemente ao uso do software ou mesmo aos estímulos em si que as populações anteriormente estudadas.

Outro ponto a ser discutido é a escolha dos estímulos apresentados aos participantes. Buscando-se garantir a boa sensibilidade do instrumento, realizou-se um estudo piloto antes do início da coleta de dados do presente estudo. Feito isso, utilizaram-se apenas estímulos com índice de reconhecimento superior a $60 \%$. Apesar desse cuidado, os resultados do estudo indicam que uma escolha mais criteriosa dos estímulos teria sido relevante. É possível que estímulos com maior índice de reconhecimento tivessem causado menos erros de identificação entre a população parkinsoniana, o que certamente alteraria o resultado estatístico deste estudo. Diante do resultado obtido, certamente conclui-se pela necessidade de aprimoramento do software e realização de estudos para sua validação. Ademais, não se utilizaram métodos de tratamento estatístico mais refinados, como a Teoria de Resposta ao Item ou análises bayesianas, pelos quais a probabilidade de respostas poderia ser analisada de forma diferenciada da estatística clássica. É importante considerar que tais métodos poderiam ter sido úteis desde o estudo piloto, na medida em que seleção de estímulos relevantes e de alta sensibilidade seriam ideais para compor o instrumento de avaliação.

Também cabe aqui referir que um diferente controle de variáveis intervenientes potencialmente poderia alterar os resultados do estudo. Por exemplo, o horário de ingestão das medicações e o registro de suas quantidades pode ter vindo a influenciar o resultado desta pesquisa, uma vez que a literatura tem indicado os possíveis efeitos do eixo dopaminérgico no reconhecimento de expressões faciais emocionais (Lawrence et al., 2007; Dujardin et al., 2004). Tal fato pode, inclusive, ter contribuído para o grupo de parkinsonianos ter tido melhor desempenho que o controle em algumas emoções, uma vez que a realização do procedimento experimental logo em seguida ao uso da medicação, potencialmente pode trazer benefício ao participante, igualando-o ao grupo normal em termos de desempenho. Sugere-se que tais fatores possam ser melhor observados em pesquisas futuras, uma vez que podem ser um fator limitador do presente estudo.

Apesar do resultado não ser significativo, é interessante observar que o TEPEF identificou menor reconhecimento para as faces de surpresa e raiva no grupo experimental em relação ao controle. Em estudos realizados com outras patologias, como a Doença de Huntigton, a qual também afeta os gânglios da base, bem como disfunção fronto-subcortical e a síndrome de Korsakoff (patologia fronto-límbica), também se observa o enfraquecimento do reconhecimento da surpresa (Montagne, Kessels, Wester, \& Hann, 2006). Clark et al. (2008) defendem que a emoção de surpresa é ambígua, pois pode ser confundida tanto com alegria (emoção de surpresa boa), como com medo (emoção de espanto). Mediante tal resultado apontado por outros estudos, é possível que uma maior amostra ou o tratamento dos dados por outros métodos estatísticos pudesse ter alcançado diferenças significativas. Entretanto, como a menor taxa de reconhecimento de surpresa e raiva não foi significativa, estudos complementares são necessários para que tal possibilidade seja esclarecida.

Tem sido discutido na literatura internacional que características específicas da DP podem influenciar o reconhecimento das emoções, já que diversas estruturas anatômicas parecem estar relacionadas a essa habilidade. Entretanto, não se estabeleceu, ainda, uma conclusão fática quanto ao assunto. Por exemplo, Cohen et al. (2010) discutem que dados conflitantes a respeito da participação dos gânglios da base no reconhecimento emocional têm sido encontrados. No caso do presente estudo, a correlação entre as variáveis específicas da DP (tempo da doença, uso de medicação específica, comprometimento motor da doença e lateralidade acometida) e o teste de reconhecimento de expressões faciais não mostrou diferenças significativas. A preocupação em relacionar essas variáveis veio dos estudos de Suzuki et al. (2006), que afirmaram ser a duração da doença, a severidade motora e o uso de medicação dopaminérgica fatores influenciadores do reconhecimento das expressões emocionais faciais na DP. Entretanto, em contraposição ao estudo citado anteriormente, neste estudo, especificamente, o bom desempenho dos parkinsonianos no teste de reconhecimento das expressões pode ter-se dado em função da baixa progressão da doença, uma vez que o grupo experimental apresentou de dois a nove anos de diagnóstico, assim como comprometimento motor leve. Tal hipótese também é discutida por Clark et al. (2008), ao abordarem a proporcionalidade do prejuízo motor em relação ao declínio cognitivo. Outra justificativa cabível para o bom desempenho dos parkinsonianos na tarefa desta pesquisa é o fato de a maioria ter ensino superior completo, o que pode ter-lhes dado maior reserva cognitiva quando comparados a pacientes com menos escolaridade. Infelizmente, este estudo não foi delineado para responder completamente a essa questão, uma vez que a escolaridade dos participantes não foi controlada. Estudos futuros pdem investigar melhor tal hipótese na população brasileira.

\section{Referências}

Adolphs, R., Schul, R., \& Tranel, D. (1998). Intact recognition of facial emotion in Parkinson's disease. Neuropsychology, 12, 253-358. doi: 10.1037/0894-4105.12.2.253

Alonso-Recio, L., Martín-Plasencia, P., Loeches-Alonso, A., \& Serrano-Rodríguez, J. (2014). Working memory and facial expression recognition in patients with Parkinson's disease. Journal of the International Neuropsychological Society, 20, 496-505. doi:10.1017/S1355617714000265 
Ariatti, A., Benuzzi, F., \& Nichelli, P. (2008). Recognition of emotions from visual and prosodic cues in Parkinson's disease. Neurology Science, 29, 219-227. doi: 10.1007/s10072-0080971-9

Assogna, F., Pontieri, F. E., Caltagirone, C., \& Spalletta, G. (2008). The recognition of facial emotion expressions in Parkinson's disease. European Neuropsychopharmacology, 18, 835-848. doi 10.1016/j.euroneuro.2008.07.004

Beck, A., Steer, R., \& Brown, G. (1996). Beck Depression Inventory. Manual of the Psychological Corporation. San Antonio: Harcourt Brace \& Company.

Borod, J. C., Welkowitz, J., Alpert, M., Brozgold, A. Z., Martin, C., Peselow, E., \& Diller, L. (1990). Parameters of emotional processing in neuropsychiatric disorders: Conceptual issues and a battery of tests. Journal of Communication Disorders, 23, 247-271. doi: 10.1016/0021-9924(90)90003-H

Breitenstein, C., Daum, I., \& Ackermann, H. (1998). Emotional processing following cortical and subcortical brain damage: Contribution of the fronto-striatal circuitry. Behavior Neurolology, 11, 29-42. doi: 10.1155/1998/579029

Chaudhuri, K.R., Healy, D.G., \& Schapira, A.H.V. (2006). Nonmotor symptoms of Parkinson's disease: Diagnosis and management. Lancet Neurology, 5, 235-245. doi: 10.1016/ S1474-4422(06)70373-8

Clark, U.S., Neargarder, S., \& Cronin-Golomb, A. (2008). Specific impairments in the recognition of emotional facial expressions in Parkinson's disease. Neuropsychologia, 46(9), 2300-2309. doi: 10.1016/j.neuropsychologia.2008.03.014

Clark, U.S., Neargarder, S., \& Cronin-Golomb, A. (2010). Visual exploration of emotional facial expressions in Parkinson's disease. Neuropsychologia, 48, 1901-1913. doi: 10.1016/j. neuropsychologia.2010.03.006

Cohen, H., Gagné, M.H., Hess, U., \& Pourcher, E. (2010). Brain and Cognition, 72, 457-463. doi: 10.1016/j.bandc.2010.01.001

Cools, R., Miyakawa, A., Sheridan, M., \& D’Esposito, M. (2010). Enhanced frontal function in Parkinson's disease. Brain, 133(1): 225-233. doi: 10.1093/brain/awp301

Costa, A., Peppe, A., Dell'Agnello, G., Carlesimo, G. A., Murri, L., Bonuccelli, U., \& Caltagirone, C. (2003). Dopaminergic modulation of visual-spatial working memory in Parkinson's disease. Dementia and Geriatric Cognitive Disorders, 15, 55-66. doi: 12566593

Dara, C., Monetta, L., \& Pell, M. D. (2008). Vocal emotion processing in Parkinson's disease: Reduced sensitivity to negative emotions. Brain Research, 1188, 100-111. doi: 10.1016/j.brainres.2007.10.034

Darwin, C. (1872/2000). A expressão das emoções nos homens e nos animais ( $2^{\mathrm{a}} \mathrm{ed}$.). São Paulo. Companhia das Letras.

De Souza, W. C., Feitosa, M. A. G., Eifuku, S., Tamura, R., \& Ono, T. (2008). Face perception in its neurobiological and social context. Psychology \& Neuroscience, 1, 15 -20. doi: 10.3922/j. psns.2008.1.003

Dujardin, K., Blairy, S., Defebvre, L., Duhem, S., Noël, Y., Hess, H., \& Destée, A. (2004). Deficits in decoding emotional facial expressions in Parkinson's disease. Neuropsychologia, 42, 239-250. doi: 10.1016/S0028-3932(03)00154-4

Ekman, P., \& Friesen, W.V. (1971). Constants across cultures in the face and emotion. Journal of Personality and Social Psychology, 17(2), 124-129. doi: 10.1037/h0030377
Ekman, P. \& Oster, H. (1979). Facial expressions of emotion. Annual Review of Psychology, 30, 527-554. doi: 10.1146/annurev. ps.30.020179.002523

Ekman, P. (1993). Facial expression and emotion. American Psychologist, 48(4), 376-379. doi: 10.1037/0003-066X.48.4.384

Fernández-Dols, J-M., (2013). Advances in the study of facial expression: An introduction to the special section. Emotion Review, 5, 3-7. doi: 10.1177/1754073912457209

Fernández-Dols, J-M., \& Crivelli, C. (2013). Emotion and expression: Naturalistic studies. Emotion Review, 5, 24-29. doi: $10.1177 / 1754073912457229$

Foltstein, M. F., Folstein, S.E., \& McHugh, P.R. (1975). Minimental state: A practical method for grading the cognitive state of patients for the clinician. Journal of Psychiatric Research, 12(3), 189-198. doi: 10.1016/0022-3956(75)90026-6

Gray, M. H., \& Tickle-Degnen, L. (2010). A meta-analysis of performance on emotion recognition tasks in parkinson's disease. Neuropsychology, 24(2), 176-191. doi: 10.1037/ a0018104

Herrera, E., Cuetos, F., \& Rodríguez-Ferreiro, J. (2011). Emotion recognition impairment in Parkinson's disease patients without dementia. Journal of the Neurological Sciences, 310, 237-240. doi: 10.1016/j.jns.2011.06.034

Hoehn, M. M. \& Yahr, M.D. (2001). Parkinsonism: Onset, progression, and mortality. Neurology, 57(10), 11-26.

Jack, R. E., Blais, C., Scheepers, C., Schyns, P. G., \& Caldara, R. (2009). Cultural confusions show that facial expressions are not universal. Current Biology, 19, 1543-1548. doi: 10.1016/j. cub.2009.07.051

Jacobs, D. H., Shuren, J., Bowers, D., \& Heilman, K.M. (1995). Emotional facial imagery, perception, and expression in Parkinson's disease. Neurology, 45, 1696-1702. doi: 10.1212/ WNL.45.9.1696

Janvin, C., Aarsland, D., Larsen, J.P., \& Hugdahl, K. (2003). Neuropsychological profile of patients with Parkinson's disease without dementia. Dementia and Geriatric Cognitive Disorders, 15, 126-131. doi: 10.1159/000068483

Kan, Y., Kawamura, M., Hasegawa, Y., Mochizuki, S., \& Nakamura, K. (2002). Recognition of emotion from facial, prosodic and written verbal stimuli in Parkinson's disease. Cortex, 38, 623630. doi: 10.1016/S0010-9452(08)70026-1

Ladislau, R. (2010). Percepção de expressões faciais emocionais em idosos com doença de Alzheimer (Dissertação de Mestrado não publicada). Universidade de Brasília, Brasília, Distrito Federal, Brasil.

Ladislau, R., Guimarães, J. G., \& De Souza, W. C. (2015). Percepção de expressões faciais emocionais em idosos com doença de Alzheimer. Psicologia Reflexão e Crítica, 28(4), 804-812. doi: 10.1590/1678-7153.201528419

Lawrence, A. D., Calder, A. J., McGowan, S. W., \& Grasby, P. M. (2002). Selective disruption of the recognition of facial expressions of anger. NeuroReport, 13, 881-884.

Lawrence, A. D., Goerendt, I. K., \& Brooks, D. J. (2007). Impaired recognition of facial expressions of anger in Parkinson's disease patients acutely withdrawn from dopamine replacement therapy. Neuropsychologia, 45, 65-74. doi: 10.1016/j. neuropsychologia.2006.04.016 
Lee, J. E., Park, H. J., Park, B., Song, S. K., Sohn, Y. H., Lee, J. D., \& Lee, P. H. (2010). A comparative analysis of cognitive profiles and white-matter alterations using voxel-based diffusion tense imaging between patients with Parkinson's disease dementia and dementia with Lewy bodies. Journal of Neurology, Neurosurgery and Psychiatry 81, 320-326. doi: 10.1136/jnnp

Martínez-Corral, M., Pagonabarraga, J., Llebaria, G., PascualSedano, B., Garcia-Sanchez, C. Gironell, A., \& Kulisevsky, J. (2010). Facial emotion recognition impairment in patients with Parkinson's disease and isolated Apathy. Parkinson `s Disease, 1, 1-5. doi: 10.4061/2010/930627

Mentis, M.J. \& Delalot, D. (2005). Depression in Parkinson's disease. In K. E. Anderson, W. J. Weiner, \& A. E. Lang (Eds.), Advances in Neurology: Behavioral neurology of movements disorders (vol. 96, pp. 26-41). Raven Press: New York. Montagne, B., Kessels, R. P. C., Wester, A. J., \& de Haan, E. H. F. (2006). Processing of emotional facial expressions in Korsakoff's syndrome. Cortex, 42(5), 705-710. doi: 10.1016/ S0010-9452(08)70408-8

Pell, M. D., \& Leonard, C. L. (2005). Facial expression decoding in early Parkinson's disease. Cognitive Brain Research, 23, 327-340. doi: 10.1016/j.cogbrainres.2004.11.004

Perón, J., Dondaine, T., Le Jeune, F., Grandjean, D., \& Vérin, M. (2012). Emotional processing in Parkinson's disease: A systematic review. Movement Disorders, 27(2), 186-199. doi: $10.1002 / \mathrm{mds} .24025$

Phillips, L. H., Channon, S., Tunstall, M., Hedenstrom, A., \& Lyons, K. (2008). The role of working memory in decoding emotions. Emotion, 8(2), 184-191. doi: 10.1037/1528-3542.8.2.184

Possin, K. L., Filoteo, J. V., Song, D. D., \& Salmon, D. P. (2008). Spatial and object working memory deficites in Parkinson's disease are due to impairment in different underlying processes. Neuropsychology, 22(5), 585-595. doi: 10.1037/a0012613

Pringsheim, T., Jette, N., Frolkis, A., \& Steeves, T.D.L. (2014). The prevalence of Parkinson's disease: A systematic review and meta-analysis. Movement Disorders, 29(13), 1583-1590. doi: $10.1002 / \mathrm{mds} .25945$

Raven, J. C. (1997). Matrizes progressivas: Escala geral. Rio de Janeiro: CEPA.

Rosso, A. L. Z., Nicaretta, D.H., \& Mattos, J.P. (2008). Correlações anatomoclínicas na doença de parkinson. Revista Brasileira de Neurologia, 44(4), 41-47.

Russel, J. A. (1994). Is there universal recognition of emotion from facial expression? A review of the cross-cultural studies. Psychological Bulletin, 115(I), 102-141. doi: 10.1037/00332909.115.1.102

Santana, C.C.V., de Souza, W.C., \& Feitosa, M.A.G. (2014). Recognition of facial emocional expressions and its correlation with cognitive abilities in children with Down syndrome. Psychology and Neuroscience, 7(2), 73-81. doi: 10.3922/j. psns.2014.017
Schröder, C., Möbes, J., Schütze, M., Szymanowski, F., Nager, W., Bangert, M., .... Dengler, R. (2006). Perception of emotional speech in parkinson's disease. Movement Disorders, 21, 17741778. doi: $10.1002 / \mathrm{mds} .21038$

Simons, G., Ellgring, H., \& Pasqualini, M. S. (2003) Disturbance of spontaneous and posed facial expressions in Parkinson's disease. Cognition and Emotion, 17(5), 759-778. doi: 10.1080/02699930302280

Sprengelmeyer, R., Young, A.W., Mahn, K., Schroeder, U., Woitalla, D., Büttner, T., ... Przuntek, H. (2003). Facial expression recognition in people with medicated and unmedicated Parkinson's disease. Neuropsychologia, 41, 1047-1057. doi: 10.1016/S0028-3932(02)00295-6

Stoffers, D., Berendsea, W. W., Deijenb, J. B., \& Wolters, E. Ch. (2003). Deficits on Corsi's block-tapping task in early stage Parkinson's disease. Parkinsonism and Related Disorders 10 (2003), 107-111. doi: 10.1016/S1353-8020(03)00106-8

Suzuki, A., Hoshino, T., Shigemasu, K., \& Kawamura, M. (2006). Disgust-specific impairment of facial expression recognition in Parkinson's disease. Brain, 129(3), 707-717. doi: 10.1093/ brain/awl011

Wieser, M.J., Mühlberger, A., Alpers, G.W., Macht, M., Ellgring, H., \& Pauli, P. (2006). Emotion processing in Parkinson's disease: Dissociation between early neuronal processing and explicit ratings. Clinical Neurophysiology, 117, 94-102. doi: 10.1016/j.clinph.2005.09.009

Yip, J. T. H., Lee, T. M. C., Ho, S-L., Tsang, K-L., \& Li, L. S. W. (2003). Emotion recognition in patients with idiopathic Parkinson's disease. Movement Disorders, 18, 1115-1122. doi: $10.1002 / \mathrm{mds} .10497$

Yoshimura, N., Kawamura, M., Masaoka, Y., \& Homma, I. (2005). The amygdala of patients with Parkinson's disease is silent in response to fearful facial expressions. Neuroscience, 131, 523-534. doi: 10.1016/j.neuroscience.2004.09.054

Xie, Y., Meng, X., Xiao, J. Zhang, J., \& Zhang, J. (2016). Cognitive changes following bilateral deep brain stimulation of subthalamic nucleus in Parkinson's disease: A metaanalysis. BioMed Research International, 2016, 1-6. doi: $10.1155 / 2016 / 3596415$

Recebido em 20.04.2016

Primeira decisão editorial em 17.10.2016

Versão final em 03.03.2017

Aceito em 13.03.2017 